\title{
LA INTEgRación ENTRE Ciencia, OPINión PÚblica Y DEMOCRACIA: APORTES DE JOHN DEWEY Y HEATHER DOUGLAS A LA DISCUSIÓN CONTEMPORÁNEA
}

The Integration of Science, Public Opinion, And Democracy: John Dewey's and Heather Douglas' CONTRIBUTIONS TO CONTEMPORARY DISCUSSION

\author{
Livio MatTARollo \\ Universidad Nacional de La Plata / CONICET, ARGENTINA \\ livio.mattarollo@nacio.unlp.edu.ar
}

\begin{abstract}
Within the debate on the value-free science ideal, the relation between scientific inquiries and citizenry has been largely considered. According to Heather Douglas, given that under certain circumstances moral and social values play a legitimate role in science, two crucial questions are what and whose values should they be. Douglas presents an analyticdeliberative model to include and promote public participation in science and claims that such a model transcends the debates over which democratic ideal should we pursue. However, considering the complexities of the very idea of democracy as well as the different ways to support the inclusion of the public into science, some extra analysis is needed. The purpose of this article is to offer a theoretical framework to Douglas' proposal by referring to John Dewey's ideas about inquiry, public opinion, and democracy. Following Dewey, it is pointed out that at a normative level scientific inquiry as a solving-problem practice and democracy as conjoint communicated experience shapes each other by means of a scientifically informed public opinion. To conclude, it is claimed that Deweyan ideas offer coherent and solid philosophical grounds to Douglas' proposal. In addition, the relevance of pragmatism for contemporary discussions on science, values and politics is highlighted.
\end{abstract}

Keywords: scientific inquiry $\bullet$ values $\bullet$ public $\bullet$ democracy $\bullet$ pragmatism

ACCEPTED: $27 / 08 / 2021$

\section{Introducción}

La relación entre investigación científica y valores, en especial aquellos tradicionalmente categorizados como extra o no-epistémicos, ha sido uno de los temas más tratados en la indagación filosófica contemporánea sobre el conocimiento científico. Dichas reflexiones toman como eje el denominado ideal de ciencia libre de valores, cuya consideración implica analizar no sólo si es posible una ciencia libre de valores sino también cómo se interpreta el ideal, qué se entiende por valores y sobre qué tipo de valores se concentra la discusión, en qué instancia inciden y/o deberían incidir, qué legitimidad tienen, si el ideal es deseable y finalmente qué consecuencias se 
siguen de asumir estas distintas perspectivas en términos de la objetividad y racionalidad de la empresa científica. Además, estas cuestiones traen consigo la discusión acerca del vínculo entre ciencia y sociedad, esto es, la discusión por qué lugar tiene o debería tener la ciudadanía al momento de tomarse algunas decisiones en la actividad científica y por cuáles serían los posibles diseños institucionales para dar curso a esa participación. Así, el debate abre múltiples frentes de consideración teórica y práctica e incluye distintas perspectivas y disciplinas.

En términos generales el ideal de ciencia libre de valores plantea que la validez de las afirmaciones científicas depende de la evidencia disponible y de una correcta derivación lógica, sin referencia a las perspectivas morales, políticas o sociales de quien investiga. En este sentido, la tarea de la ciencia remitiría a los hechos, un dominio autónomo y totalmente independiente del plano de los valores o preferencias (Kincaid et al. 2007, p.4). De acuerdo con Reisch (2009), y más allá de que sus raíces históricas puedan rastrearse siglos atrás, la formulación contemporánea del ideal de ciencia libre de valores se origina durante la Guerra Fría a partir de la forma profesional, apolítica y técnica que adopta la filosofía de la ciencia de Estados Unidos de la segunda mitad del siglo XX en función de elaborar una legitimación filosófica de las pretensiones científico-militares del gobierno de ese país. Ya hacia la década de 1980, la reformulación progresiva de la distinción entre contextos de justificación / descubrimiento y una visión más precisa de lo que implica la práctica científica hizo que los límites del ideal se volvieran más definidos. Dicho de otra forma, si bien se reconoció ampliamente que la ciencia apela a valores no-epistémicos en su dimensión "externa", se restringió aún más su fortaleza "interna".

Uno de los partícipes más reconocidos del debate es Hugh Lacey. De acuerdo con Lacey, hay presencia efectiva y legítima de valores al momento de adoptar estrategias que ofrecen el marco o dominio para construir teorías. En esta línea, Lacey \& Mariconda (2014, pp.182-85) elaboran un modelo de interacción entre actividad científica y valores por el cual no sólo hay lugar legítimo para valores morales y sociales en la etapa de adopción de estrategias (etapa $\mathrm{M}_{1}$ ) sino en el desarrollo de la investigación $\left(\mathrm{M}_{2}\right)$, difusión de resultados $\left(\mathrm{M}_{4}\right)$ y aplicación del conocimiento científico $\left(\mathrm{M}_{5}\right)$. Sin embargo, no debería permitirse la incidencia de valores morales y sociales en la etapa de validación cognitiva de teorías $\left(\mathrm{M}_{3}\right)$ dado que esta última tiene como criterio de evaluación y horizonte regulativo el ideal de imparcialidad. Dicho ideal admite exclusivamente valores epistémicos o cognitivos y justifica la incidencia de valores sociales y morales en $\mathrm{M}_{1}$ y $\mathrm{M}_{2}$ sólo en cuanto contribuyen causalmente a su obtención (Lacey 1997, pp.25-27; Lacey \& Mariconda 2014, pp.181-83). En este sentido, se acentúa la distinción entre los niveles lógicos y metodológicos de la selección de estrategias o el desarrollo de la investigación, por un lado, y la estricta elección de teorías, por el otro. ${ }^{1}$ Más aún, la única diferencia entre el ideal de ciencia libre de valores y el modelo de interacción es la inclusión de una función legítima de valores 
morales y sociales en $\mathrm{M}_{1}$ (Lacey \& Mariconda 2014, p.186). Así, señala Ricardo J. Gómez, más allá de cierta apertura en la adopción de estrategias, la posición de Lacey representaría una rigorización de la versión tradicional de la neutralidad valorativa de la ciencia entendida como exclusión de valores no-epistémicos en el contexto de justificación (2014, p.170).

El ideal de ciencia libre de valores en general y la posición de Lacey en particular han dado lugar a numerosos análisis críticos. Pueden identificarse discusiones desde enfoques feministas (Anderson 1995, 2004; Longino 1990), desde la identificación del pluralismo teleológico y axiológico de las prácticas científicas (Olivé 2000; Echeverría 2002) pero también posiciones que proponen distintas alternativas como el ideal de ciencia bien ordenada (Kitcher 2001; 2011), de ciencia guiada por valores sociales (Longino 2002) o de ciencia socialmente responsable (Kourany 2010). Por su parte, Heather Douglas (2000; 2007; 2009; 2014; 2016, entre otros) considera que el ideal en términos de neutralidad, imparcialidad y autonomía como criterio para la evaluación de las prácticas científicas no sólo es inalcanzable [unattainable] e insostenible [untenable] sino sobre todo un mal ideal que en ciertos casos conduce a una visión inaceptable de la ciencia. Como contrapartida, afirma que en determinadas circunstancias los valores no-epistémicos son necesarios para un buen razonamiento en ciencia (Douglas 2007, pp.121-22).

Una primera aproximación al pensamiento de Douglas permite advertir que tematiza dos aspectos centrales del debate: (i) el rol de los valores no-epistémicos en la actividad científica y (ii) los vínculos entre ciencia y política, a efectos de proponer diseños institucionales para la participación ciudadana en ciertas instancias de las investigaciones. Dicho en pocas palabras, Douglas considera que es necesario desarrollar una ciencia públicamente responsable de sus juicios de valor y del modo en que sopesa los efectos de los posibles errores. Ahora bien, si la evaluación de las consecuencias extra-epistémicas mediante valores sociales y morales se torna indispensable, surge la pregunta por cuáles y de quiénes son los valores empleados para ese análisis, pregunta que según la autora incluye consideraciones acerca de la democracia misma (Douglas 2005). Además de reconocer que hay distintos modelos de democracia que involucran diversos estándares de participación del público en algunas decisiones de la actividad científica, Douglas sugiere que su propio enfoque trasciende los debates sobre el ideal de democracia que debería seguirse. Así, parecería que simplemente asume o supone que la participación del público requiere un modelo democrático pero no desarrolla el tema con el grado de detalle que presentan sus argumentos sobre la dimensión netamente epistemológica, dejando de lado una discusión de enorme complejidad y centralidad para su proyecto.

Frente a este cuadro, intentaré dar razones para fundamentar la participación del público en ciertos planos de la actividad científica a partir de un sentido preciso de democracia al que dicha participación queda normativamente vinculada. Para ello 
recurriré al enfoque del pragmatista clásico John Dewey, en particular a su definición de democracia como experiencia conjunta comunicada basada en la consolidación de un núcleo de valores compartidos por los distintos grupos sociales (1998) y a sus nociones de público y de opinión pública (2004). Así, intentaré señalar que Dewey ofrece un marco teórico apropiado no sólo para pensar el vínculo entre ciencia y democracia sino también para articular y sostener la propuesta específica de Douglas sobre el modelo de participación analítico-deliberativa del público en ciencia. En este sentido, prosigo una línea esbozada por Cuevas Badallo y Urueña López (2019) aunque me concentro en la dimensión valorativa del vínculo entre ciencia, opinión pública y democracia a fines de considerar dicho modelo institucional. Para ello, en la segunda sección recuperaré los argumentos de Douglas sobre la inconveniencia e incluso imposibilidad de separar taxativamente el plano científico del político; en la tercera sección me detendré en el enfoque deweyano a fines de aclarar cómo se concibe el vínculo entre ciencia y democracia en cuanto mediado por la opinión pública; finalmente, en la cuarta sección evaluaré la propuesta de Douglas en favor de señalar en qué puntos encontraría apoyo en el desarrollo de Dewey. En suma, buscaré brindar un marco filosófico al planteo de Douglas e intentaré enfatizar la vigencia de los aportes deweyanos al momento de reflexionar en torno al necesario vínculo entre ciencia, valores y política.

\section{Ciencia, valores y el espectro de la democracia}

Antes de avanzar en el objetivo previsto resulta necesario reconstruir brevemente algunos aspectos teóricos sobre el rol que asigna Douglas a los valores no-epistémicos en la investigación científica. Douglas interpreta el ideal en términos de valores aceptados y no aceptados en el proceso de inferencia o validación, es decir, como un "ideal de emplear sólo valores epistémicos en la inferencia científica" (Douglas 2016, p.3). ${ }^{2}$ Frente al ideal así comprendido, su planteo podría articularse en dos instancias: una pars destruens de crítica y rechazo del ideal; y una pars construens con su propuesta de una nueva estructura de valores en ciencia. En cuanto a la primera, Douglas "desafía" al ideal desde tres puntos de vista: (i) descriptivo; (ii) de los límites — dada la porosidad entre los valores epistémicos y no-epistémicos por la influencia del contexto de las investigaciones; y (iii) normativo — dados el argumento del vacío [gap], vinculado con la tesis de la sub-determinación de la teoría por la evidencia disponible, y el argumento del error, relacionado con el riesgo inductivo de toda investigación (Douglas 2016; sobre los argumentos del desafío normativo, Brown 2013). Desde aquí afirma que cuando la ponderación del riesgo inductivo requiere la consideración de las consecuencias extra-epistémicas de la investigación, dicha ponderación incluye legítimamente valores no-epistémicos en cada uno de los niveles internos de 
la ciencia en función de determinar los riesgos que se asumen o las decisiones que se toman:

[...] los valores no epistémicos son una parte necesaria de los aspectos internos del razonamiento científico para casos en los que el riesgo inductivo incluye el riesgo de consecuencias no epistémicas. En estos casos, la ciencia libre de valores es una ciencia inadecuada; el razonamiento es defectuoso e incompleto. Por lo tanto, el estándar normativo debe ser reconsiderado. Para la ciencia que tiene claros impactos no epistémicos, ser 'libre de valores' no es un objetivo loable. (Douglas 2000, pp.559-60).

Una vez establecido este diagnóstico, Douglas propone una estructura de valores en ciencia tal que permita distinguir la incidencia directa e indirecta de valores cognitivos, sociales y morales intervinientes en la actividad científica, sea que actúen como razones que determinan por sí mismos las decisiones en el curso de la investigación o que actúen para sopesar la importancia de la incertidumbre con relación a la afirmación que se analiza o a la decisión que se toma, respectivamente. Así, la incidencia de valores sociales y morales es legítima en la medida en que operen de modo indirecto, esto es, para evaluar las consecuencias del error y la suficiencia de la evidencia. Por el contrario, el rol directo de este tipo de valores daría lugar a una ciencia "politizada" que pierde su integridad y pone en cuestión la confianza en el juicio científico (Douglas 2008, pp.11-13; 2009, pp.87-114; 2015). El enfoque de Douglas ha recibido algunas objeciones acerca de la ambigüedad entre el carácter lógico o consecuencialista de la distinción de roles (Elliott 2011, 2013), acerca de la posibilidad de que el rol indirecto de valores no-epistémicos arroje resultados indeseados y acerca de la dificultad de explicar algunas decisiones de las investigaciones apelando a la referida distinción de roles (Steel \& Whyte 2012). ${ }^{3}$ No obstante, esta posición resulta muy interesante al momento de considerar el lugar de los valores en ciencia tanto a nivel descriptivo como normativo y, además, constituye un punto de apoyo para sus elaboraciones sobre ciencia y política.

He señalado anteriormente que uno de los desafíos que Douglas eleva al ideal es el desafío de los límites. Junto con lo dicho sobre la porosidad entre tipos de valores, ese desafío incluye una segunda consideración, a saber: el cuestionamiento de la distinción procedimental entre "evaluación del riesgo" y "manejo del riesgo", pensada para defender cierta integridad de la ciencia y distinguir las funciones de la ciencia y la política en algunas decisiones. En efecto, toda investigación y en especial aquellas orientadas al diseño o asesoramiento de políticas públicas requiere evaluar la evidencia pero también las consecuencias extra-epistémicas de los posibles errores; sólo así el razonamiento es completo y quienes investigan cumplen con sus responsabilidades morales (Douglas 2004, p.238). Ahora bien, dado que ese análisis (i) se realiza en el curso de la investigación y no a su término; (ii) conlleva toma de decisiones en cuanto a la metodología, la recolección de datos, la caracterización de evidencia, etc. 
que reorientan la propia investigación; y dado también que (iii) las responsabilidades de rol de quienes investigan no anulan ni suplantan sus responsabilidades generales, entonces las fronteras entre evaluación y manejo del riesgo se tornan permeables y la distinción entre ciencia y política como ámbitos exentos o plenos de valores, respectivamente, pierde su sentido (sobre la distinción de responsabilidades, Douglas 2009 pp.71-79; 2014). En suma, "[c]ualquier límite, ontológico, procedimental, u otro, que aspire a separar a la ciencia de la política sobre la base de la presencia de valores en la política o la ausencia de valores en ciencia está condenado a fracasar." (Douglas 2004, p.240).

Si las investigaciones empleadas para el asesoramiento de políticas públicas requieren la consideración de sus consecuencias extra-epistémicas por parte de quienes investigan, dado el riesgo inductivo endémico, ¿qué estrategias deben adoptarse, pues, para que esa consideración fuera legítima? En primer lugar, la apelación a valores morales y sociales debe respetar siempre el rol indirecto, esto es, debe intervenir en la ponderación de suficiencia de evidencia y en el análisis de las consecuencias potenciales pero nunca debe fungir como razones por sí mismas. En segundo lugar, y a diferencia de lo que suele suceder en los papers o demás publicaciones especializadas, se deben explicitar las resoluciones en base a valores de modo que el público y los funcionarios políticos puedan advertir la estructura valorativa de la investigación y puedan iniciar una discusión al respecto. En tercer lugar, Douglas parece sugerir que sería saludable contar con cierto consenso general en torno a algunos valores que sustente las evaluaciones de la investigación: "Si puede lograrse acuerdo social sobre qué valores deberían predominar, o sobre qué valores frecuentemente en tensión debería apoyarse la ponderación (como salud pública versus productividad industrial), los(as) científicos(as) pueden emplear ese consenso para guiar los juicios de valor que configuran su trabajo y sus interpretaciones." (Douglas 2004, p.239). ${ }^{4}$

En virtud de lo dicho resulta claro que Douglas reserva un lugar legítimo para valores sociales y morales en la dimensión "interna" de la actividad científica. Resulta claro, también, que inscribe a las investigaciones en un contexto más amplio del que difícilmente puedan abstraerse, a menos que mantengamos la ficción del aislacionismo de la ciencia (Douglas 2005, p.155). En este sentido, una cualidad central del enfoque de Douglas - a diferencia, por ejemplo, del modelo de interacción entre actividad científica y valores de Lacey \& Mariconda- radica en la identificación de roles legítimos para los valores morales y sociales en todas las etapas de la actividad científica. Así las cosas, surge una cuestión crucial, ya adelantada en la introducción: ¿cuáles y de quiénes son los valores que deben tenerse en cuenta? Lo dicho respecto del acuerdo social aporta algunas pistas. Sin embargo, Douglas va más allá del consenso y bajo la idea de que no es aceptable que ninguna élite minoritaria imponga sus valores señala que estas preguntas "invocan el espectro de asuntos y cuestiones democráticas [raises the specter of the democratic concerns]" (2005, p.156). 
En este punto nos encontramos con el concepto mismo de democracia, tan complejo como disputado. Sin pretensiones de exhaustividad vale señalar que se ha distinguido entre democracia directa y democracia representativa, democracia formal y sustancial, democracia como regla de la mayoría, como mecanismo deliberativo y democracia consensual (Bobbio 2005, pp.441 y ss.). También se ha diferenciado entre democracia política, social y económica o industrial (Sartori 1993, pp.5-8). Desde otro punto de vista, se han distinguido formas de democracia liberal en sus variantes utilitaristas, elitistas y pluralistas (o de grupos), tanto como modos de democracia social o participativa en sus teorías de la soberanía, perfeccionistas o dialógicas (Nino 1997, pp.101-53). Las aristas del asunto se multiplican si se considera la relación entre público, ciencia y democracia: no sólo se debe precisar qué noción de democracia está involucrada sino también, indica la propia Douglas, qué comprensión de la ciencia y qué niveles de educación científica tiene el público (Douglas 2017). Todo ello entra en juego a la hora de evaluar distintos diseños institucionales de participación ciudadana en ciertas decisiones concernientes a la actividad científica. Al respecto, se ha argumentado que los diversos mecanismos de participación encuentran su mejor funcionamiento y sus mejores criterios de evaluación en relación con aquellas teorías de la democracia que enfatizan la participación directa (Fiorino 1990). También se ha propuesto la modalidad de análisis participatorio, que se pretende adecuada tanto para teorías de participación directa como para teorías pluralistas o de participación de grupos (Laird 1993). Más recientemente se han identificado distintas correlaciones entre concepciones de ciencia, modelos de comunicación y rol del público, desde una función pasiva vinculada a la noción clásica de ciencia hasta la idea del públicoactor en el modelo de ciencia ciudadana (Cuevas Badallo y Urueña López 2019) y se han elaborado distintos modelos de participación relacional co-constructivista (Chilvers y Kearnes 2020). Más allá de la valiosa discusión sobre distintas concepciones de ciencia y de la recopilación de propuestas de diseño institucional -puntos que no abordaré en esta oportunidad- me interesa rescatar un diagnóstico crítico: en los estudios del área CTS habría cierta tendencia a importar categorías de "democracia" y "público" predadas, fijas y externas al diseño de prácticas de participación ciudadana, común a distintos modelos teóricos de democracia como el deliberativo y el agonista (Chilvers y Kearnes 2020, pp.352-53).

Por su parte, Douglas señala que su propuesta de modelos de deliberación analítico-colaborativa está apoyada en una concepción filosófica del conocimiento científico y que pretende trascender los debates sobre qué ideal de democracia debería perseguirse (2005, p.153). Sin embargo, el brevísimo panorama recién presentado permite advertir que su elaboración requeriría algunas precisiones, tanto acerca de qué entiende por democracia como de las articulaciones entre esta última y el modelo de participación pública en ciencia que sugiere. En este punto propongo recuperar las reflexiones John Dewey en torno a las ideas de investigación, opinión pública y de- 
mocracia a efectos de ofrecer una reconstrucción teórica que oficie de marco para el argumento de Douglas. Esta referencia obedece a ciertas afinidades conceptuales de ambos planteos así como a la aclaración de Douglas — bastante marginal, en una breve nota- de que su idea de "insertar al público en la ciencia" recupera el concepto de público del pragmatista (2005, p.168, n. 1). En lo que sigue me abocaré al enfoque de Dewey sobre democracia, público e investigación, siempre bajo la idea de que es relevante y consistente con la perspectiva de Douglas.

\section{Configuraciones recíprocas entre ciencia, opinión pública y democracia}

Como es bien sabido, en el marco de la filosofía de Dewey pensar en términos políticos es pensar en términos de democracia. Por supuesto, abordar todas las dimensiones de la perspectiva deweyana sobre el tema excede el recorte del presente estudio pues ello requeriría analizar, cuanto menos, sus fundamentos filosóficos y su impacto en los distintos temas de reflexión del pragmatista (ética, educación, arte, etc.). Sin embargo, introduciré algunas observaciones para enmarcar el planteo. De acuerdo con Honneth (1999), y visto que la preocupación de Dewey por la democracia es transversal a toda su obra, es posible identificar al menos dos grandes períodos en su tratamiento del tema. El primero abarca desde la década de 1880 hasta la de 1910 y se basa en el individualismo de la libertad, la responsabilidad y la iniciativa, a efectos de elaborar una justificación ética de la democracia. El segundo, desde la década de 1910 en adelante, se centra en la esfera pública, sin omitir reflexiones sobre el individuo. ${ }^{5}$

En este segundo período Dewey establece con claridad que la idea de democracia admite dos grandes niveles, uno descriptivo o de facto y uno normativo o de jure. Así, en sus textos pueden rastrearse diversos sentidos del concepto, entre ellos y como mínimo (i) la democracia como forma de gobierno; (ii) la democracia como forma de vida; y (iii) la democracia como ideal de vida comunitaria. El primer significado se corresponde con aquella versión de la democracia sintetizada en los modos ordinarios de elección, sufragio y representación que dan cuerpo a la regla de la mayoría. Sin embargo, Dewey no demora en advertir que "[u]na democracia es más que una forma de gobierno; es primariamente un modo de vivir asociado, de experiencia conjunta comunicada." (Dewey 1998, p.82. MW.9.93. Traducción levemente modificada). ${ }^{6} \mathrm{El}$ advenimiento de la democracia como forma de vida requiere el reconocimiento por parte de los distintos grupos sociales de un núcleo de valores comunes y estables. Para que ello suceda Dewey estipula (i) que al interior del grupo debe haber numerosos y variados intereses y valores conscientemente compartidos y libremente comunicados; y (ii) que entre los grupos debe haber una interacción 
plena que propicie el enriquecimiento recíproco de sus intereses y que conduzca a un reajuste permanente debido a las nuevas situaciones producidas por el múltiple intercambio (Dewey 1998, pp.80-81. MW.9.89-92). Así, la auténtica comunidad se define por la existencia de un interés común articulado a través de la comunicación democrática, comunicación que potencia tanto a los grupos como a los individuos que los integran pues hace que los intereses particulares devengan intereses socializados y compartidos (Festenstein 1995, p.177).

Asimismo, en su inconcluso y relativamente poco atendido proyecto de filosofía social Dewey ofrece un criterio ideal de comparación que enfatiza esta idea de enriquecimiento mutuo de los grupos sociales: "[p]odemos elaborar en nuestra imaginación una imagen en la que haya desarrollo equitativo y proporcionado de todas las formas de vida asociada, en la que éstas interactúen libremente entre sí, y en la que los resultados de cada una contribuyan al enriquecimiento e importancia de las otras [...]" (Dewey 2015, p.16. Traducción propia). Resulta especialmente interesante señalar que Dewey identifica a las organizaciones científicas como uno de los grupos del entramado social, nucleado en torno a actividades, propósitos, necesidades e intereses comunes como la investigación, el descubrimiento y el desarrollo intelectual (Dewey, MW.15.236 y 2015, p.16). Por tanto, si para alcanzar el modo de vida democrático los intereses y valores de los grupos sociales no deberían divergir radicalmente y si las organizaciones científicas constituyen un grupo social determinado, sus intereses y valores deberían estar en línea con los del resto de la sociedad en favor de un desarrollo equilibrado. ${ }^{7}$ Tal es el punto de apoyo de la democracia como ideal de vida comunitaria, conformada no sólo por la actividad asociada o conjunta - tal es una condición necesaria pero no suficiente- sino por la conciencia de un tipo de organización cuyas actividades conjuntas son promovidas en tanto y en cuanto sus consecuencias se evalúan como beneficiosas por todas las personas directa e indirectamente involucradas. En este marco se obtiene un desarrollo individual y grupal integrado pues las influencias e intereses de los distintos grupos se reforzarían mutuamente y sus valores tenderían a coincidir como resultado de la deliberación libre y sistemática (Dewey 2004, p.138. LW.2.328). ${ }^{8}$

Hay otros aspectos sobre el vínculo entre ciencia y democracia que requieren atención. El primero, que mencionaré brevemente, se vincula con el modelo metodológico que ofrece la ciencia para la resolución de problemas sociales. La investigación controlada supone voluntad para investigar, examinar y obtener conclusiones. Supone también considerar la diversidad de opiniones, indagar libre y abiertamente, sujetar toda conclusión a futuras revisiones y promover la crítica pública. En los asuntos públicos este modelo permitiría orientar los esfuerzos para reemplazar intereses de clase, prejuicios sociales, raciales, nacionales o incluso el peso de la tradición. Así, "[... ] el futuro de la democracia está aliado con la difusión de la actitud científica. Es la única garantía contra la desorientación general por medio de la propaganda. Más 
importante aún, es la única manera de asegurar la posibilidad de formar una opinión pública lo bastante inteligente para enfrentarse a los problemas sociales." (Dewey 1965, p.149. LW.13.168. Cursivas agregadas)..$^{9}$

El segundo punto se desprende inmediatamente del anterior y refiere al doble vínculo entre opinión pública y conocimiento científico, en la medida en que la ciencia es una actividad pública y por lo tanto es objeto de regulación por parte de la opinión pública pero a la vez la ciencia informa y en ese sentido constituye a la opinión pública. Para avanzar en estas observaciones resulta indispensable recuperar dos elementos. En primer lugar, la investigación en general y la investigación científica en especial puede ser definida como resolución de problemas, según explicita Dewey en Lógica. Teoría de la investigación: "[1]a investigación es la transformación controlada o dirigida de una situación indeterminada en otra que es tan determinada en sus distinciones y relaciones constitutivas que convierte los elementos de la situación original en un todo unificado." (Dewey 1950, p.123. LW.12.108). Estas observaciones establecen con claridad el carácter eminentemente práctico de la investigación. Las investigadoras no observan el mundo sino que toda investigación es dentro del mundo, dirigida y deliberada, con la intención de cambiar una situación confusa e insegura por otra clara y resuelta. En este sentido, la idea deweyana de investigación es lo suficientemente amplia para incluir la situación inicial definida como problemática pero también la instancia de instrumentación o "regreso" al curso ordinario de experiencia. Luego, lejos de ser una empresa orientada a la representación, el conocimiento en general y el conocimiento científico en particular se orienta a la modificación del material existencial, de acuerdo con los propósitos adelantados en la investigación. Finalmente, la validez de la investigación incluye la consideración de las conclusiones de amplio alcance como constitutiva de la investigación, más allá de las distintas instancias que identifica el pragmatista en la pauta o matriz general de la investigación:

[d]el mismo modo como no es posible determinar la validez de una proposición en el raciocinio o, en general, del material conceptual, prescindiendo de las consecuencias a que da origen su empleo funcional, tampoco se puede determinar la garantía suficiente de un juicio que pretende ser conocimiento (en su sentido laudatorio) con independencia de su conexión con un amplio círculo de consecuencias. [...] Aunque la concordancia entre las actividades científicas y las consecuencias que provocan en el público en general (técnicamente no científico) se hallan en un plano diferente, sin embargo, esa concordancia constituye una parte integrante de la prueba completa de las conclusiones físicas, siempre que sus repercusiones públicas son de significación (Dewey, 1950, p.537. LW.12.483-84. Cursivas agregadas).

En segundo lugar, Dewey ofrece una particular distinción entre acciones públicas y privadas que estructura su posición sobre el asunto aquí tratado (2004, pp.64-65. 
LW.2.243-44). Del conjunto de acciones de los seres humanos es posible identificar dos grandes tipos de consecuencias: aquellas que afectan sólo a las personas directamente involucradas en la transacción inicial y aquellas que afectan a otras personas distintas de las directamente involucradas en la transacción inicial. Así, la amplitud y el alcance de las consecuencias de las transacciones definirán si las mismas son privadas, en el primer caso, o públicas, en el segundo. Desde aquí se sigue, por un lado, que el alcance de las consecuencias de las acciones determina si estas últimas son tan importantes como para ser controladas y reguladas mediante su restricción o promoción. Por otro lado, que aquellas personas que logran reconocerse como afectadas, para bien o para mal, por las consecuencias indirectas de determinadas acciones públicas y que consecuentemente tienen un interés conjunto en controlar esas consecuencias conforman lo que Dewey denomina un público. Los públicos no son organizaciones estancas sino que varían con la misma dinámica de las acciones públicas y de los intereses y necesidades que éstas originen, al tiempo que una misma persona puede estar involucrada en distintos públicos en función de las formas en que se vea afectada por distintas acciones públicas. En este orden, la opinión pública es el juicio que forman quienes componen el público respecto de los asuntos públicos. La opinión pública se erige entonces como un elemento central en cuanto articula la orientación epistemológica del procedimiento democrático con la concepción de una comunidad constituida en torno a ciertos valores compartidos y deviene el medio discursivo para la solución cooperativa de problemas bajo condiciones democráticas (Honneth 1999, p.97). ${ }^{10}$

Sobre la base de esta distinción y dado el alcance de sus consecuencias, resulta evidente que la actividad científica es eminentemente pública y que por tanto debería tener regulación por parte del público afectado. Si se considera que la prueba completa de la validez de la investigación incluye la ponderación del amplio círculo de consecuencias y que la investigación científica efectivamente genera un amplio círculo de consecuencias, el criterio formulado por Dewey establece la necesidad de incluir legítimamente valoraciones morales, sociales y políticas en el curso de la investigación e introduce una nueva razón para incrementar la participación pública en algunas decisiones de las investigaciones. Más aún, este aspecto de la relación entre público y ciencia pone de relieve la importancia de otra pregunta: si la investigación es resolución de problemas ¿de quiénes son los problemas que habrán de resolver las investigaciones científicas?

La relación entre ciencia y opinión pública incluye otra nueva consideración acerca del modo en que la primera constituye a la segunda. En el marco del debate entre Dewey y Walter Lippmann sobre el rol de los expertos, el pragmatista cuestiona las alternativas que limitan la circulación de información y la toma de decisiones a círculos restringidos porque hace del conocimiento científico una herramienta para la consolidación de las clases dominantes y aleja a la ciencia de los intereses y asun- 
tos públicos, en los términos anteriormente establecidos. Esto lo conduce a afirmar que "[1] a clase de expertos se encuentra tan inevitablemente alejada de los intereses comunes que se convierte en una clase con unos intereses privados y un conocimiento privado que en cuestiones sociales no es conocimiento en modo alguno." (2004, p.168. LW.2.364). ${ }^{11}$ Así, el gobierno de expertos(as) sería una alternativa que obtura el surgimiento y crecimiento de cualquier idea proveniente de otro campo porque no permite instancias de debate, consulta o persuasión y evita que se conviertan en ideas de dominio público. En efecto, propicia el monopolio de la posesión del conocimiento y de la toma de decisiones, esto es, fortalece a un sector determinado cuyos fines desvinculados del resto de los grupos sociales se tornan contrarios al sentido más amplio y profundo de la democracia (Dewey 2004, p.168. LW.2.364).

A diferencia de ello, y como señala del Castillo (2004, p.27), cuando Dewey establece la relación entre ciencia e investigación social libre [free social inquiry] quiere decir que los intereses y necesidades del público son los elementos últimos de trabajo de la ciencia y al mismo tiempo que la opinión pública debe nutrirse del conocimiento científico especializado. Esto permite comprender por qué las críticas recién presentadas de ninguna manera implican desestimar el valor de la experticia de quienes investigan porque cuanto más precisas sean las investigaciones, más precisos serán los juicios evaluativos en los que se apoyan las valoraciones propiamente dichas. ${ }^{12}$ Sin embargo, la pericia técnica no garantiza que el conocimiento científico quede vinculado al interés público sino que para ello es indispensable que la ciencia se aplique en [in] los asuntos humanos y devenga conocimiento en el sentido honorable y categórico del concepto, observa Dewey (2004, p.151. LW.2.344). Así, no obstante la necesidad de la experticia técnica de las investigadoras debe tenerse en cuenta la necesidad de mejorar los métodos y condiciones de comunicación de los resultados de las investigaciones para que fueran empleados en la resolución inteligente de asuntos públicos. De manera esquemática, si para Dewey el público son todas aquellas personas que se reconocen afectadas por las consecuencias indirectas de las transacciones y la forma de garantizar el reconocimiento es la plena publicidad de todos los asuntos referentes al público, entonces la comunicación es condición para la constitución del público que se organiza políticamente y regula las consecuencias indirectas de las diversas acciones. Si, además, se asume que la ciencia es una actividad pública, entonces la comunicación también es una condición que se aplica a la investigación científica:

En todo lo dicho queda implícito que el conocimiento es comunicación además de comprensión. [...] Un hecho de la vida en comunidad que no se difunda hasta ser un dominio común es una contradicción en los términos. [... L La comunicación de los resultados de la investigación social es lo mismo que la formación de la opinión pública. [... Sólo la investigación continua, o sea, una investigación interconectada y reiterada, puede proporcionar el 
material de una opinión duradera sobre los asuntos. [... ] Desde luego, puede darse una honesta divergencia en cuanto a las políticas que hay que emprender, incluso cuando los planes surgen del conocimiento de los mismos hechos. Pero la política auténticamente pública no se puede generar a menos que esté informada por el conocimiento, y este conocimiento no existe excepto cuando hay investigación e información sistemática, exhaustiva y bien provista. (Dewey, 2004, pp.152-53. LW.2.345-46).

A partir de estas consideraciones queda claro el rol que debería tener la investigación en la formación de la opinión pública. Dewey busca consolidar un público con competencias democráticas tal que garanticen una interpretación crítica, reflexiva e inteligente no solo de los datos ofrecidos por las investigaciones sino y especialmente de su importancia y relevancia [bearing] para resolver o dirimir asuntos públicos (DeCesare 2012, p.113). En este sentido, la investigación científica adquiere un valor político deseable y por derecho propio dentro del proyecto deweyano porque permite hacer del ciudadano en particular y de los distintos públicos en general actores competentes para participar en la esfera pública, a fin de determinar los asuntos que deba tratar - entre ellos, algunos vinculados a las amplias consecuencias de investigaciones científicas particulares. Teniendo en cuenta lo dicho, en sus sentidos más plenos la investigación científica y la democracia como experiencia conjunta comunicada se configuran recíprocamente, tanto a nivel metodológico como del conocimiento necesario para la deliberación.

\section{Algunos aportes al modelo de deliberación analítico-colaborativa}

Como he indicado anteriormente, existe una amplia gama de propuestas de diseño institucional para la participación ciudadana en ciencia. Dada esta variedad, Douglas propone un criterio general de evaluación: la medida de interacción e influencia mutua entre público y expertos(as). Este criterio daría lugar a diseños que superen dos situaciones frecuentes, aquella en la que ciudadanos y expertos tienen poca o nula interacción y aquella en la que se desarrolla un proceso deliberativo profundo pero sin impacto ulterior en las decisiones de los expertos (2004, p.157). Para que ocurra dicha intensificación de la interacción e influencia, la comunicación de la ciencia no debe limitarse a que el público simplemente acepte las afirmaciones científicas sino que debe fortalecer el carácter recíproco del vínculo tal que incentive la participación del público y permita una crítica legítima de la actividad científica (2017, p.84). Douglas también propone algunas pautas más concretas de interacción entre ciencia y público mediante el modelo de deliberación analítico-colaborativa, al que me dedicaré a continuación. 
En mi lectura, uno de los aspectos más novedosos e interesantes del enfoque de Douglas es la centralidad que otorga a la deliberación en base a valores. En efecto, reconoce como eje de la interacción entre ciencia y público las diversas disputas en torno de aquellos valores que legítimamente pueden tener lugar en la actividad científica. Douglas identifica dos grandes momentos en los que generalmente se suscitan esas disputas: la definición de la agenda de investigación y las instancias de validación del conocimiento, esto es, la referida consideración de la suficiencia de evidencia a la luz del análisis de las consecuencias no-epistémicas del posible error. ${ }^{13}$ Además, recupera distintos casos para ilustrar cada una de las instancias, algunos de alcance local y otros de alcance nacional. Un primer ejemplo sobre la disputa en la agenda de investigación es el registro de las diferencias de criterios de científicos(as) y madres en torno a efectos secundarios poco frecuentes de ciertas vacunas (criterios de gran escala y criterios individualizados, respectivamente), diferencias que en algunos casos han sido punto de partida para posiciones antivacunas. Un segundo ejemplo es la determinación del nivel admisible de utilización de neonicotinoides dada la muerte masiva en colonias de abejas luego de la aplicación de pesticidas en campos y granjas (Douglas 2017, pp.91-94). Más allá de estos ejemplos, recupera algunos casos de participación ciudadana que adoptan el modelo de deliberación analíticocolaborativa, entre ellos la investigación en torno al tipo de barcos remolcadores que deberían utilizarse en el comercio marítimo de petróleo en Alaska a fines de evitar derrames de producto, la investigación sobre los modos de eliminación de armas químicas en Estados Unidos o sobre las posibles soluciones ante la contaminación de napas de agua potable y del propio río Columbia producto de la producción de plutonio en Hanford, Washington (Douglas 2005, pp.159-62). En estos casos las ventajas identificadas por Douglas son tres: (i) el público puede ayudar a definir y encuadrar mejor el problema de investigación; (ii) el público puede aportar conocimiento relevante acerca de las condiciones y prácticas específicas y/o locales de la investigación; y (iii) el público puede incidir en la consideración de qué valores deben orientar o dar forma al análisis (2005, p.158).

Más allá de los grados de éxito que Douglas adscribe a cada uno de los casos en función del criterio por ella misma elaborado, me interesa explicitar los puntos en que la formulación general del modelo de deliberación analítico-colaborativa encuentra respaldo teórico en algunos de los argumentos ofrecidos por Dewey, teniendo en cuenta que tanto el pragmatista como Douglas comparten el diagnóstico sobre la necesidad de considerar las consecuencias extra-epistémicas de las investigaciones tanto como de incrementar el grado de interacción entre expertos(as) y ciudadanos(as) en el marco de una democracia que apele a la ciencia para el diseño de políticas públicas. En efecto, los ejemplos recuperados por Douglas subrayan el rol de la intervención constructiva del público y la centralidad que adquiere la explicitación de los valores subyacentes a cada alternativa en cuestión. También ilustran la 
necesidad de que las opiniones del público estén científicamente informadas pues de lo contrario serían tan (poco) relevantes como las expresiones personales de valor de los expertos (Douglas 2005, p.167). Aquí cobran relevancia las consideraciones de Dewey sobre la relación entre opinión pública y conocimiento científico respecto de la legítima regulación por parte del público, dadas las consecuencias de las investigaciones, y respecto de la relevancia del conocimiento científico para la opinión pública, a fin de que dicha regulación fuera lo más precisa y ajustada posible. Dicho en otros términos, "[1]a ciencia sin opinión pública es ideología; la opinión pública sin ciencia o es una masa, o un fantasma." (del Castillo, 2004, p.27).

Asimismo, vale recordar que Dewey pone especial énfasis en comprender el modo de vida democrático por referencia a la integración de los intereses de los grupos sociales, integración que ofrecería criterios generales para tomar y evaluar ciertas decisiones de carácter valorativo en el curso de la investigación o, como plantea Douglas, ofrecería cierto consenso respecto de qué valores deberían predominar para guiar esas decisiones. En este sentido, los criterios que plantea Dewey se definen en términos de valores, incluso teniendo en cuenta que la participación del público adopta distintas modalidades. No se trata de pensar una participación "directa" del público para evaluar, por ejemplo, la suficiencia de la evidencia de efectividad de las distintas vacunas contra el Covid-19 en adultos-mayores. Se trata, en cambio, de que quienes investigan tengan en cuenta los consensos generales en torno a cómo se valora la vida de esos adultos-mayores, qué protección se les brinda, cómo se balancean los riesgos de efectos secundarios adversos ante la urgencia de los contagios masivos, etc. Por último, y tomando algunas expresiones de Douglas, Dewey parece asumir la porosidad de los límites entre los ámbitos de la ciencia y la política en un plano descriptivo, dado que dicha porosidad efectivamente no se mantiene (la pretensión de lograrlo resulta muchas veces una forma de legitimar intereses privados), y también en un plano normativo pues bajo las condiciones antedichas la investigación científica tiene un rol fundamental en la consolidación de los distintos públicos y consecuentemente de la democracia.

Más allá de lo señalado, Dewey y Douglas difieren respecto a su comprensión de la actividad científica: si para el primero la investigación se comprende como una práctica de resolución de problemas, para la segunda "[1]a ciencia es una empresa basada en la evidencia que busca construir un conocimiento empírico del mundo. Lo hace mediante la propuesta de teorías explicativas que incluyen evidencia disponible y el subsiguiente testeo de esas teorías para potenciar su capacidad [to the best of its ability]." (Douglas 2017, p.86). Esta definición parece vincularse con las concepciones más clásicas del conocimiento, en términos de explicación empírica del mundo, y reproduce una visión estrecha de la investigación como elaboración de teorías. Asimismo, deja por fuera las instancias de definición del objeto de la investigación y de la metodología, en un movimiento que parecería contrario a su pretensión de dis- 
cutir con las versiones "aislacionistas" de la ciencia y que parecería justificar el rol indirecto de los valores sociales y morales por default, es decir, por la falta de evidencia total para sostener los juicios científicos. En mi interpretación, la concepción amplia de investigación de Dewey articula con mayor claridad el vínculo entre investigación y contexto experiencial, por un lado, e investigación y público, por el otro. Desde esta perspectiva toda investigación se inicia con una situación indeterminada definida como problemática, lo cual incluye deliberación y valoración. Además, el conocimiento científico adquiere su sentido pleno en la medida en que fuera aplicado en los asuntos públicos, aquellos que el público defina como problemáticos y necesarios de resolver. Finalmente, la prueba completa de la investigación requiere la evaluación de las consecuencias de amplio alcance, en especial si afectan significativamente al público, evaluación que por supuesto incluirá valores tradicionalmente denominados no-epistémicos en cuanto constitutivos de dicho sentido amplio de investigación.

En este punto vale aclarar lo siguiente: lejos de cualquier reduccionismo cientificista, la deliberación pública científicamente informada siempre es de corte político pues si bien la información provista por la ciencia es relevante para resolver problemas comunes, su regulación y más precisamente la pregunta por sus fines debe incluir una consideración acerca de la consistencia de esos mismos fines con el núcleo de valores e intereses compartidos por la comunidad. En efecto, "[... ] la prueba que sirve para decidir si un supuesto bien es auténtico o espurio nos la proporciona su capacidad de resistir la publicidad [publicity] y la comunicación." (Dewey 1993, p.210. MW.12.197). ${ }^{14}$ Aquí las posiciones de Dewey y Douglas se acercan nuevamente pues coinciden en que se torna indispensable explicitar los valores subyacentes a las orientaciones generales pero también a las decisiones particulares de las investigaciones, a fines de abrir la discusión sobre los valores involucrados, en caso de que fuera necesario. En palabras de Douglas, "[s]i los(as) científicos(as) pueden hacer esos juicios en privado, sin explicitarlos en sus publicaciones, y así dar forma a una política pública sin lugar para la rendición de cuentas pública, todo estándar de democracia habrá sido violado." (Douglas 2005, p.156). La publicidad y comunicación son entonces garantías frente a cierta tendencia a privatizar los intereses y fines de la investigación. Puesto en otros términos, son garantías de que los valores que intervienen legítimamente en la investigación sean aquellos que los distintos grupos sociales reconocen como comunes en el marco de la siempre abierta deliberación democrática. En esta misma línea Douglas indica que "[... ] la ciencia será un lugar de debate y crítica en la medida en que sus implicancias sean relevantes para el público y para el diseño de políticas. La ciencia no es un mecanismo para producir acuerdos o unanimidad en el público. La ciencia es un recurso para el debate y un recurso para imaginar nuestro futuro en el ya complejo ámbito público.” (2017, p.94). Debido a estas razones, y aun cuando Douglas indique que su pretensión es trascender las discusiones acerca del modelo de democracia que debería seguirse, considero que 
el enfoque de Dewey ofrece argumentos consistentes con las ideas centrales de la filósofa para vincular normativamente a la investigación científica y a la participación pública con un sentido determinado de democracia y de ideal de vida comunitaria.

\section{Conclusiones}

El recorrido propuesto ha estado guiado por dos cuestiones centrales, ambas derivadas del planteo de Douglas en torno a los diseños institucionales para propiciar la participación ciudadana en ciencia. Asumida la legítima incidencia indirecta de valores sociales y morales en determinadas instancias de la investigación científica, surge la pregunta por cuáles y de quiénes son los valores que intervienen. Si bien Douglas pretende enfocarse en el asunto de la participación ciudadana en ciencia y trascender las discusiones sobre modelos de democracia, la complejidad de esta última noción tanto como de sus vínculos teóricos y prácticos con la actividad científica ponen de relieve la necesidad de precisar algunas aristas del asunto. Así las cosas, he acudido a algunas ideas de Dewey sobre investigación científica, opinión pública y democracia a efectos de ofrecer un marco teórico para la propuesta de Douglas capaz de explicitar de forma articulada y fructífera los vínculos antedichos.

Las referencias a Dewey han estado concentradas en tres aspectos. Por un lado, he puntualizado en su definición amplia de investigación en cuanto resolución de una situación indeterminada definida como problemática. Esta concepción permite plantear la pregunta por quiénes definen a la situación como problemática, que se suma a las identificadas por Douglas. Por otro lado, he recuperado la idea deweyana de democracia como forma de vida o experiencia conjunta comunicada en cuanto caracterizada por la constitución de un núcleo de valores compartidos por todos los grupos sociales, núcleo que ofrece una primera guía al momento de considerar cuáles son los valores sociales y morales que podrían intervenir en la actividad científica. Finalmente, he enfatizado que de acuerdo con Dewey los sentidos más plenos de ciencia y democracia se constituyen recíprocamente, siendo la opinión pública un mediador fundamental entre ambos. Es por ello que Dewey alienta la experticia técnica sin recostarse en posiciones cientificistas o tecnocráticas. Al contrario, frente a la tendencia de cierta clase de expertos a conducir las investigaciones en favor del beneficio privado, la comunicación y la puesta en discusión pública y política de los fines de la investigación deviene un elemento indispensable para la democracia y para el proyecto de Gran Comunidad.

En virtud de lo dicho, considero que estas ideas de Dewey constituyen un aporte relevante tanto a la propuesta de Douglas como, en términos más generales, a la discusión sobre ciencia y política en el marco del debate sobre el ideal de ciencia libre de valores. En efecto, su noción amplia de investigación en términos de acción trans- 
formacional del curso de experiencia incluye la determinación del problema tanto como la valoración de las consecuencias de amplio alcance en cuanto aspectos que constituyen la prueba completa de su validez — algo que no parecería suceder con la definición de ciencia de Douglas, más vinculada a una concepción tradicional del conocimiento como representación y explicación. Además, el modo en que Dewey vincula investigación científica y democracia hace que su propuesta de participación del público no corra el riesgo de "importar" categorías para analizar el vínculo entre ciencia, opinión pública y democracia sino que ofrece una perspectiva consistente para responder por qué se requieren entre sí. En suma, aun cuando Dewey no avance en la propuesta de diseños institucionales de participación del público en ciencia, como bien hace Douglas, sus ideas parecen brindar respuestas a las preguntas por quiénes definen las situaciones como problemáticas (es decir, la agenda de investigación), qué valores deberían incluirse en caso necesario (como mínimo, aquellos concordantes con el núcleo de valores compartidos y susceptibles de plena comunicación y publicidad) y de quiénes son esos valores (del público debidamente constituido e informado).

Para finalizar me interesa dejar planteados algunos breves comentarios. En primer lugar, es indudable que el enfoque de Douglas incluye aspectos de suma relevancia tanto para discutir el ideal de ciencia libre de valores como, y especialmente, para proponer alternativas que den cuenta del funcionamiento de la investigación y corran por la senda de la filosofía política de la ciencia. En segundo lugar, la consideración de las consecuencias no epistémicas de las investigaciones científicas contribuye a inscribirlas en contextos de análisis más amplios y a complejizar las consideraciones filosóficas sobre la actividad científica. En tercer lugar, el esfuerzo de proponer diseños institucionales en línea con el desarrollo teórico antedicho y con el concepto deweyano de público hacen que la perspectiva de Douglas contemple distintos frentes de argumentación y ofrezca promisorias líneas de trabajo. A estos aportes podrían agregarse las referidas contribuciones del enfoque de Dewey, que ayudan a robustecer el planteo general de Douglas y que, en esa misma medida, dan cuenta de la vigencia del pensamiento del pragmatista al momento de reflexionar sobre la necesaria integración entre investigación científica, valores y política.

\section{Referencias}

Anderson, E. 1995. Knowledge, Human Interests, and Objectivity in Feminist Epistemology. Philosophical Topics 23(2): 27-58.

Anderson, E. 2004. Uses of Value Judgments in Science: A General Argument, with Lessons from a Case Study of Feminist Research on Divorce. Hypatia 19(2): 2-24.

Bernstein, R. 2010. Filosofía y democracia: John Dewey. Trad. R. del Castillo. Barcelona: Herder. 
Bobbio, N. 2005. Democracia. En N. Bobbio, N. Matteucci \& G. Pasquino (ed.), Diccionario de Política, p.441-453. Trad. J Aricó, M. Soler y J. Tula . México DF: Siglo XXI.

Brown, M. 2013. Values in Science. Beyond Underdetermination and Inductive Risk. Philosophy of Science 80(5): 829-839.

Chilvers, J., \& Kearnes, M. 2020. Remaking Participation in Science and Democracy. Science, Technology, \& Human Values 45(3): 347-380.

Cuevas Badallo, A. 2008. Conocimiento científico, ciudadanía y democracia. Revista CTS. Ciencia, Tecnología y Sociedad 10(4): 67-83.

Cuevas Badallo, A. \& Urueña López, S. 2019. Públicos y actores en la democratización de la actividad científica. Revista CTS 42(14): 9-29.

DeCesare, T. 2012. The Lippmann-Dewey "debate" revisited: the problem of knowledge and the role of experts in modern democratic theory. Philosophical Studies in Education 43: 106-116. del Castillo, R. 2004. Érase una vez en América. En John Dewey. La opinión pública y sus problemas [1927], Trad. R. del Castillo, p.11-55. Madrid: Morata.

Dewey, J. The Collected Works of John Dewey, 1882-1953 (1967-1987). The Early Works, 18821898 (1967-1972); The Middle Works, 1899-1924 (1976-1983); The Later Works, 19251953 (1981-1991). Carbondale: Southern Illinois University Press.

Dewey, J. 1950 [1938]. Lógica. Teoría de la Investigación. Trad. E. Imaz. México: FCE.

Dewey, J. Syllabus. Social Institutions and the Study of Morals. The Collected Works of John Dewey. Carbondale: Southern Illinois University Press. The Middle Works, Vol. 15, p.229_ 73.

Dewey, J. 1965 [1939]. Libertad y cultura. Trad. R. Castillo Dibildox. México: Unión Tipográfica Editorial Hispano-Americana.

Dewey, J. 1998 [1916]. Democracia y Educación. Trad. L. Luzuriaga. Madrid: Morata.

Dewey, J. 2004 [1927]. La opinión pública y sus problemas. Trad. R. del Castillo. Madrid: Morata.

Dewey, J. 2008 [1939]. Teoría de la valoración. Un debate con el positivismo sobre la dicotomía de hechos y valores. Trad. A. Di Berardino y Á. Faerna. Madrid: Biblioteca Nueva.

Dewey, J. 2015 [1919-1921]. Lectures in social and political philosophy. European Journal of Pragmatism and American Philosophy VII(2): http://journals.openedition.org/ejpap/404. Access: 10/03/2020.

Douglas, H. 2000. Inductive Risk and Values in Science. Philosophy of Science 67(4): 559-579.

Douglas, H. 2004. Border Skirmishes Between Science and Policy: Autonomy, Responsibility, and Values. En P. Machamer \& G. Wolters (Eds.), Science, Values and Objectivity, p.220244. Pittsburgh: Pittsburgh University Press.

Douglas, H. 2005. Inserting the Public into Science. En S. Maasen \& P. Weingart (Eds.), Democratization of Expertise? Exploring Novel Forms of Scientific Advice in Political DecisionMaking, p.153-169. Nederland: Springer.

Douglas, H. 2008. The Role of Values in Expert Reasoning. Public Affairs Quarterly 22(1): $1-18$.

Douglas, H. 2009. Science, Policy and the Free-Value Ideal. Pittsburgh: University of Pittsburgh Press.

Douglas, H. 2014. The Moral Terrain of Science. Erkenntnis 79(S5): 961-979.

Douglas, H. 2015. Politics and Science: Untangling Values, Ideologies, and Reasons. The Annals of the American Academy of Political and Social Science 658: 296-306. 
Douglas, H. 2016. Values in Science. En P. Humphreys (ed.), The Oxford Handbook of Philosophy of Science, p.609-630. New York: Oxford University Press.

Douglas, H. 2017. Science, Values and Citizens. En M. P. Adams et. al. (eds.), Eppur si muove: Doing History and Philosophy of Science with Peter Machamer. A Collection of Essays in Honor of Peter Machamer, pp.83-96. Springer (E-book).

Echeverría, J. 2002. Ciencia y valores. Barcelona: Ediciones Destino.

Elliott, K. 2011. Direct and Indirect Roles for Values in Science. Philosophy of Science 78(2): 303-324.

Elliott, K. 2013. Douglas on Values: From Indirect Roles to Multiple Goals. Studies in History and Philosophy of Science 44(3): 375-383.

Festenstein, M. I. 1995. Pragmatism and Liberalism: Interpreting Dewey's Political Philosophy. Res Publica 1(2): 163-182.

Festenstein, M. I. 2019. Does Dewey have an "Epistemic Argument" for Democracy? Pragmatism Today (2-3): 217-241.

Fiorino, D. 1990. Citizen Participation and Environmental Risk: A Survey of Institutional Mechanisms. Science, Technology, \& Human Values 15(2): 226-243.

Frega, R. 2015. John Dewey's Social Philosophy. A Restatement. European Journal of Pragmatism and American Philosophy VII(2): http://journals.openedition.org/ejpap/410. Access: $12 / 03 / 2021$

Gómez, R. J. 2014. La dimensión valorativa de las ciencias. Hacia una filosofía política. Bernal: Universidad Nacional de Quilmes.

Hernández, N. 2017. Expertos en la vida pública: ¿élites independientes o investigación socializada? Una aportación del debate Lippmann-Dewey en el contexto contemporáneo. Península XII(2): 169-199.

Honneth, A. 1999. Democracia como cooperación reflexiva. Trad. C. Rendón \& S. Muñoz. Estudios Políticos 15: 81-106.

Kitcher, P. 2001. Science, Truth, and Democracy. Oxford; New York: Oxford University Press.

Kitcher, P. 2011. Science in a Democratic Society. Oxford: Oxford University Press.

Kourany, J. A. 2010. Philosophy of science after feminism. Oxford: Oxford University Press.

Lacey, H. 1997. The Constitutive Values of Science. Principia 1(1): 3-40.

Lacey, H. 1999. Is Science Value Free? Values and Scientific Understanding. London \& New York: Routledge.

Lacey, H. \& Mariconda, P. R. 2014. O modelo da interação entre as atividades científicas e os valores na interpretação das práticas científicas contemporâneas. Estudos Avançados 28(82): 181-199.

Laird, F. 1993. Participatory Analysis, Democracy and Technological Decision Making. Science, Technology, \& Human Values 18(3): 341-361.

Longino, H. E. 1990. Science as Social Knowledge. Princeton, N.J: Princeton University Press. Longino, H. E. 2002. The Fate of Knowledge. Princeton, N. J.: Princeton University Press.

Midtgarden, T. 2012. Critical Pragmatism: Dewey's Social Philosophy Revisited. European Journal of Social Theory 15(4): 505-521.

Misak, C. J. 2013. The American Pragmatists. Oxford: Oxford University Press.

Nino, C. 1997. La constitución de la democracia deliberativa. Trad. R. Saba. Barcelona: Gedisa. Olivé, L. 2000. El bien, el mal y la razón. México DF: Paidós. 
Reisch, G. A. 2009. Cómo la Guerra Fría transformó la filosofía de la ciencia: Hacia las heladas laderas de la lógica. Trad. D. Blanco. Bernal: Universidad Nacional de Quilmes.

Putnam, H. 1992. A Reconsideration of Deweyan Democracy. En Renewing Philosophy, pp.180202. Cambridge: Harvard University Press.

Sartori, G. 1993. ¿Qué es la democracia? Trad. M. Gonzáles Rodríguez \& S. Pestillini. México: Editorial Patria.

Schudson, M. 2008. “The 'Lippmann-Dewey Debate' and the Invention of Walter Lippmann as an Anti-Democrat: 1986-1996". International Journal of Communications 2: 1031-1042.

Steel, D. \& Whyte, K. P. 2012. Environmental Justice, Values, and Scientific Expertise. Kennedy Institute of Ethics Journal 22(2): 163-182.

Wang, J. C. 2007. John Dewey in China: To teach and to learn. Albany: State University of New York Press.

Westbrook, R. B. 1993. John Dewey and American Democracy. Ithaca: Cornell University Press.

\section{Notas}

1 "Una teoría es adecuadamente aceptada (racionalmente considerada como encapsulando las posibilidades de un dominio) sólo si manifiesta los valores cognitivos en un alto grado y de acuerdo con el más alto estándar para evaluar su manifestación en teorías. Los valores, aquellos que hacen a esas posibilidades interesantes y que pueden motivar la consideración provisional de teorías que se adecúan a las estrategias están dialécticamente vinculados con ellas, no juegan ningún rol (adecuado) en los juicios de aceptación. Ellos no (deberían) operar junto con los valores cognitivos. La distinción y separación de momentos [en la investigación] es metodológica y lógicamente esencial." (Lacey 1999, p.227. Traducción propia). “Una teoría es adecuadamente aceptada (racionalmente considerada como encapsulando las posibilidades de un dominio) sólo si manifiesta los valores cognitivos en un alto grado y de acuerdo con el más alto estándar para evaluar su manifestación en teorías. Los valores, aquellos que hacen a esas posibilidades interesantes y que pueden motivar la consideración provisional de teorías que se adecúan a las estrategias están dialécticamente vinculados con ellas, no juegan ningún rol (adecuado) en los juicios de aceptación. Ellos no (deberían) operar junto con los valores cognitivos. La distinción y separación de momentos [en la investigación] es metodológica y lógicamente esencial." (Lacey 1999, p.227. Traducción propia).

${ }^{2}$ Todas las citas a Douglas tienen traducción propia. Cuando la traducción traiga dificultades, incluyo el fragmento original para contrastar. He decidido traducir scientist por "científico(a)" dado que Douglas suele utilizar el pronombre she para referir a a scientist: "For example, when a scientist decides to pursue a particular project because she is interested in the subject, her interest in the subject is a value judgement that serves in a direct role for the decision to pursue it. She pursues a project because of her values." (Douglas 2016, p.675. Cursivas agregadas).

${ }^{3}$ Douglas $(2016,676-77)$ señala que la distinción es principalmente de orden lógico pero como una de las principales dimensiones de la evaluación de suficiencia de evidencia es cuánta incertidumbre se acepta y ello queda vinculado con los riesgos potenciales del error, ambos sentidos están estrechamente conectados. Por otro lado, también señala que siempre es necesario conocer qué valores concretos entran en juego y que en ningún momento 
plantea que la sola distinción de roles no alcanza para explicar decisiones, tal como parecen cuestionar Steel \& Whyte (2012).

${ }^{4}$ Douglas también señala que el consenso es clave para fortalecer la confianza de la ciudadanía en la actividad científica, especialmente en torno a problemas como el cambio climático. En sus palabras, "[s]i los expertos no comparten tus valores en lo que cuenta como riesgo aceptable, pueden ser expertos a quienes se debería escuchar pero no expertos en cuya evaluación de la evidencia querrías confiar para tomar una decisión." (Douglas 2016, p.304).

${ }^{5}$ Por supuesto, esta distinción no es taxativa. Tanto Westbrook (1993, p.293) como el mismo Honneth (1999, p.94) coinciden en señalar que las conferencias previas a Naturaleza Humana y Conducta (1922) constituyen un puente entre ambos períodos en la medida en que Dewey pretende allí identificar un fundamento psicológico a su propia posición política y arguye que si los hábitos son funciones sociales entonces pueden fungir como punto de partida para la acción inteligente cooperativa y comunal.

${ }^{6}$ Las citas y referencias a los textos de John Dewey son a las versiones en español, cuando las hubiera. Como es habitual en la literatura especializada, la referencia a la traducción estará acompañada por la referencia a la edición canónica de las obras completas: The Collected Works of John Dewey 1882-1953 (1967-1987). Así, se indicará EW (The Early Works), MW (The Middle Works) o LW (The Later Works), seguido del correspondiente número de volumen y página. La única excepción se dará con el texto "Lectures in Social and Political Philosophy", editado en 2015 y no incluido en The Collected Works.

${ }^{7}$ Esto no quiere decir que Dewey no contemple las tensiones y disputas de intereses entre los distintos grupos. En efecto, su proyecto de filosofía social pragmatista incluía una teoría del conflicto social. Más allá de algunos esbozos en los documentos de clases y en La reconstrucción de la filosofía, el grueso de estas ideas aparece en sus Lectures in China (1919-1921), donde señala que los grupos sociales se nuclean en torno a necesidades básicas. Hacia 1926 Dewey parece haber abandonado definitivamente dicho proyecto. Sobre el viaje de Dewey a China, ver Wang (2007); sobre la filosofía social de Dewey, ver Midtgarden (2012) y Frega (2015), entre otros.

${ }^{8}$ Sobre el desarrollo del individuo en la comunidad, ver Festenstein (1995, pp.167 y ss.). Para una interpretación del fundamento metafísico de la democracia como ideal, ver Westbrook (1993, pp.361-66).

${ }^{9}$ Esta propuesta da lugar a la interpretación de Putnam (1992) y de Honneth (1999) sobre una justificación epistémica de la democracia bajo la forma de reflexión cooperativa comunitaria. Esta interpretación es cuestionada por Misak (2013, pp.136-38), entre otras. Para un detallado estado de la cuestión, ver Festenstein (2019, sec. 1).

${ }^{10}$ Por supuesto, en muchos casos las personas no logran reconocerse como afectadas por una acción pública y no pueden constituirse como un público propiamente dicho - de hecho, este diagnóstico de público perdido o eclipsado es el punto de partida del análisis del pragmatista (ver Dewey 2004, Cap. IV).

${ }^{11}$ Las referencias explícitas e implícitas a las obras de Lippmann junto con las reseñas que publica Dewey en The New Republic son algunas de las fuentes del denominado "Debate Lippmann-Dewey". Sin embargo, se ha sostenido que el "debate" no es sino una serie de referencias indirectas, que deberían incluirse otros actores entre los que Dewey habría hecho más bien el papel de mediador (Cuevas Badallo 2008) o que la contraposición taxativa entre Dewey y Lippmann ha sido construida a posteriori (Schudson 2008). Para una revisión del 
debate, Hernández (2017). Para una reconstrucción de las ideas centrales de Lippmann, ver Westbrook (1993, pp.294-300).

${ }^{12}$ En su teoría de la valoración Dewey distingue entre juicios evaluativos y valorativos. Los primeros se vinculan con la capacidad de prever cursos de acción en función de los elementos involucrados — de allí que se planteen en términos de condiciones-consecuencias y que requieran del conocimiento científico- mientras que los segundos se vinculan con la evaluación de valores, es decir, con la valoración en su sentido más pleno (Dewey 2008, pp.101 y ss. También Bernstein 2010, pp.151-64).

${ }^{13}$ Puesto en los términos del modelo de interacción entre actividad científica y valores de Lacey \& Mariconda (2014), el modelo de deliberación de Douglas referiría a las etapas $\mathrm{M}_{1} \mathrm{y}$ $\mathrm{M}_{2}$ pero también a $\mathrm{M}_{3}$. Asimismo, teniendo en cuenta que para Douglas no hay una distinción radical entre evaluación y manejo del riesgo, tampoco podría pensarse una diferencia tan profunda entre validación y aplicación del conocimiento sobre la base de la ausencia o presencia de valores morales, sociales y políticos, de modo que su modelo de deliberación, al incluir la deliberación sobre agenda y suficiencia de evidencia, incluiría $\mathrm{M}_{5}$ y plausiblemente $\mathrm{M}_{4}$.

${ }^{14} \mathrm{~A}$ grandes rasgos el término publicity remite al acto o hecho de hacer pública una intención, idea, interés, etc. Sin embargo, señala del Castillo, Dewey utiliza publicity en distintos sentidos, desde "libertad de expresión / discusión" o "procesos de persuasión, convicción, discusión e intercambio de ideas" hasta "formas de desarrollar confianza mutua". Así, publicity no se restringe a la ausencia de restricciones para la expresión individual sino a la "construcción de un proceso deliberado" de formación de ideas y valores (del Castillo 2004, p.23). 